\title{
Nordic Nutrition Recommendations 2004, based on scientific evidence
}

\author{
Wulf Becker
}

Information and Nutrition Department, Swedish National Food Administration, Uppsala, Sweden

\begin{abstract}
The fourth edition of the Nordic Nutrition Recommendations (NNR) includes detailed recommendations on daily physical activity. For adults $30 \mathrm{~min}$ of daily physical activity of moderate intensity and/or vigorous intensity is recommended. More physical activity (about $60 \mathrm{~min}$ daily) may be needed for prevention of weight gain. For children a minimum of $60 \mathrm{~min}$ of physical activity every day is recommended. As in the third edition, a limitation in the intake of saturated plus trans fatty acids to about $10 \%$ of the total energy intake $(E \%)$ and in the total fat intake to $30 \mathrm{E} \%$ is recommended. The intake of carbohydrate and dietary fibre intake should increase, while the intake of refined sugar should not exceed $10 E^{0} \%$. NNR are primarily intended for planning of diets for groups of healthy subjects with normal body weight and sedentary to active lifestyle. NNR are not intended for weight reduction, but give the prerequisites for the prevention of weight gain and a reduction of risk for the most common diet-associated diseases in the Nordic countries.
\end{abstract}

Keywords: chronic diseases; minerals; Nordic countries; nutrition recommendations; physical activity; vitamins

\section{Introduction}

The Nordic countries have for several decades collaborated in setting guidelines for dietary composition and recommended intakes of nutrients. Since the late 1970s this work has been carried out within the co-operation of the Nordic Council of Ministers. The first edition of the Nordic Nutrition Recommendations (NNR) was published in 1980. The fourth edition was recently approved and the main recommendations presented in this journal (1). The full report, with the background and scientific basis, is published by the Nordic Council of Ministers (2).

\section{Aims of the Nordic Nutrition Recommendations}

NNR are primarily valid for planning of diets for groups of healthy individuals with normal body weight with a physical activity corresponding to a sedentary or active lifestyle. For individuals with disease and for groups with special needs, e.g. weight reduction, the dietary composition and physical activity level may have to be adjusted accordingly.

\section{News in the fourth edition 2004}

Recommendations on daily physical activity are now included and physical activity has been taken into account for the individual nutrient recommendations wherever appropriate. For children a minimum of $60 \mathrm{~min}$ of physical activity every day is recommended. The activity can probably be divided into shorter intervals of physical activity during the course of the day. Activities should be as diverse as possible to provide optimal opportunities for developing all aspects of physical fitness.

For adults $30 \mathrm{~min}$ of daily physical activity of moderate intensity and/or vigorous intensity is recommended. This should be in addition to the energy expenditure through normal inactive living. The activity can probably be divided into shorter intervals of physical activity during the course of the day, e.g. intervals lasting for about $10 \mathrm{~min}$. More physical activity (about $60 \mathrm{~min}$ daily) of a moderate and/or vigorous intensity may be needed for prevention of weight gain.

Other changes include an increase in the recommended daily intake of vitamin $\mathrm{D}$, vitamin $\mathrm{C}$ and folate $(1,2)$. 


\section{As before}

As in the third edition, a limitation in the intake of saturated plus trans fatty acids to about $10 \%$ of the total energy intake $(E \%)$ and of the total fat intake to $30 \mathrm{E} \%$ is recommended. Intake of polyunsaturated fatty acids should be $5-10 E \%$, including 1 $E \%$ from n-3 fatty acids. The intake of carbohydrate and dietary fibre intake should increase, while the intake of refined sugars should not exceed $10 \mathrm{E} \%$. A limitation in the intake of sodium (as salt) and alcohol is recommended.

\section{Food-based dietary guidelines}

NNR also deal with the practical implementation into foods and meals, although no quantitative guidelines generally are given. For the Swedish situation the new NNR do not motivate any major changes to the recently developed guidelines (3).

\section{Basis for the Nordic Nutrition Recommendations}

The basis for setting recommendations is defined for each individual nutrient using available scientific evidence. Various research methods are available when investigating nutrient, diet and health relationships. Experimental and observational studies in humans constitute the basis both for determining human nutrient requirements, diet and health relationships, and for dietary guidelines. Few studies, involving a limited number of subjects, are available in which clinical symptoms have been induced. These are mainly classical depletionrepletion studies of vitamins. Several studies have investigated biochemical indicators of status in normal subjects fed diets with graded levels of nutrients well above the amounts necessary to induce clinical symptoms. Population studies, in which biochemical indicators of nutrient intake have been assessed and related to clinical or biochemical status, have been used in the absence of controlled studies. For some nutrients, e.g. minerals, balance studies or a factorial approach have been used to estimate requirement and recommended intake. In many cases reference values for children have been extrapolated from studies on adults.

In addition to the basic requirements for nutrients to maintain body stores and functions, NNR take into account the effects of dietary intakes and diet composition on various health outcomes. In terms of nutrients this applies mainly to the energy- providing nutrients, e.g. fatty acid composition, but also to other components such as dietary fibre and to micronutrients such as sodium, potassium and some vitamins. The studies on which the recommendations are based include dietary intervention studies with defined diets, prospective cohort studies and case-control studies. Population studies linking a health outcome to dietary intake or a biochemical marker of intake have also been evaluated. The term "health outcome" includes clinical endpoints such as coronary heart disease and markers of increased risk for disease, e.g. serum cholesterol, insulin resistance, elevated blood pressure or bone density.

When assessing nutritional requirements and the health impact of dietary changes, it is essential that both the doses of a specific nutrient and the total diet be objectively assessed. Hence, a major criterion for the studies used as evidence in NNR is that the total dietary intake during the study period is adequate for the subjects and that the intake of the component being studied is adequately measured. Another criterion is that the study period is sufficiently long for the selected indicators or endpoints, based on available knowledge. In general, stronger evidence is available from studies that involve biochemical markers of disease risk than from studies where clinical endpoints have been included. This is due to the difficulties in performing fully controlled dietary intervention studies as such studies require a large number of subjects and a long duration to obtain statistically measurable effects. Thus, the recommended intakes for those nutrients that are primarily based on diet-heath relationships in many cases have to rely on a combined evaluation of evidence from different types of study.

Several controlled intervention studies have shown that a shift to a diet in line with the NNR recommendations, e.g. about $30 \mathrm{E} \%$ fat, less than 10 $E \%$ saturated fatty acids, high in dietary fibre, with adequate amounts of n-3 fatty acids, rich in fruit, vegetables and wholemeal cereal products, and with regular consumption of fish, alone or in combination with regular physical activity, can reduce the risk and a number of risk factors for diabetes and cardiovascular disease $(1,4-8)$.

\section{Popular diets}

A range of dietary programmes or concepts has been promoted and marketed by researchers or 


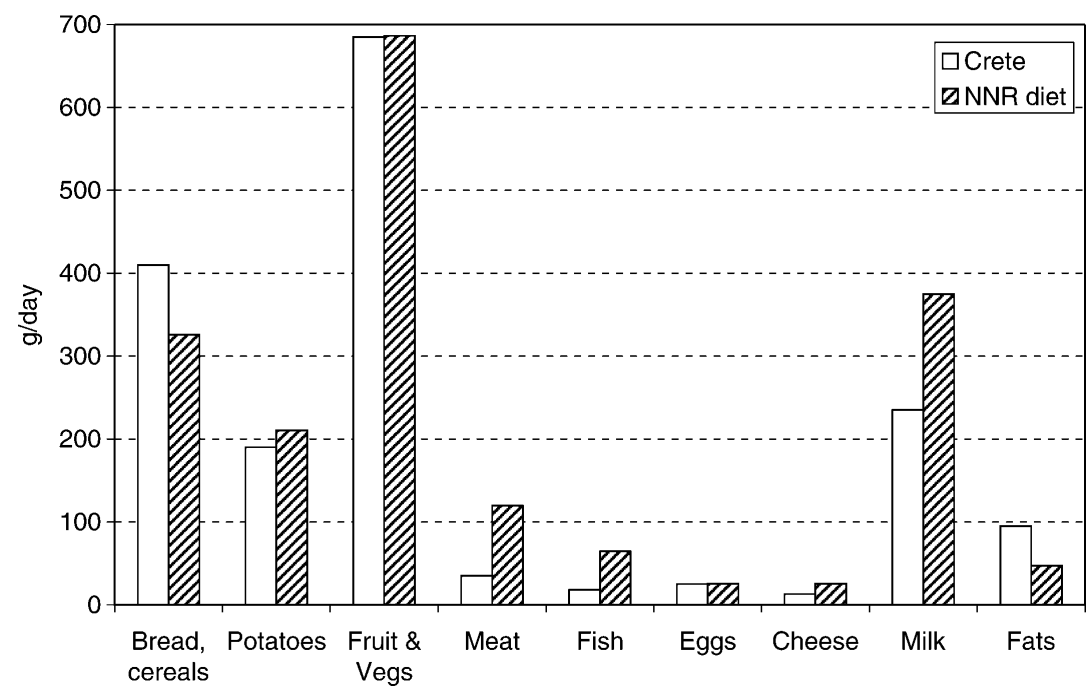

Fig. 1. Food consumption among Cretan men in the seven-country study (11) and based on the Nordic Nutrition Recommendations for a male reference subject (3).

proponents. These include diets primarily aimed at weight reduction, such as Atkins and Montignac, and to some extent methods based on the glycaemic index concept. Common to these are the relative lack of scientific evidence supporting a greater benefit compared with weight-reducing diets based on reduced-fat and high-fibre diets (9). A practical consequence of several of these methods is a reduction in the consumption of energy-dense foods high in sugar and/or fat, resulting in a reduced overall energy intake, at least in the short term.

Other dietary models such as the traditional Mediterranean diet pyramid and the alternative American Food Pyramid (Willett's food pyramid) (10) have been developed mainly based on results of epidemiological research. These two pyramids have several common features, but in the Mediterranean version potato is a staple food together with cereals, while it is categorized together with sugar, sweets and white bread in the alternative American pyramid. The latter categorization is mainly based on the glycaemic properties of potato (10). In fact, the traditional Cretan diet contained amounts of potatoes (150-200 g per day) comparable to those consumed in many Nordic countries today. A comparison of the food-based guidelines developed for the Swedish adult population with the traditional Cretan diet as described in the seven-country study (11) reveals many similarities (Fig. 1). Common features include an emphasis on fruit and vegetables, bread and cereals, and potatoes. The NNR diet includes somewhat more milk, fish and meat. In the Cretan diet olive oil was the main edible fat, whereas different types of edible fat, e.g. vegetable-based spreads, and various vegetable oils, e.g. rapeseed oil, are suggested in the NNR diet.

In summary, a body of evidence supports the view that a diet based on NNR will contribute to a reduction in risk for the most common dietassociated diseases in the Nordic countries, such as cardiovascular disease, obesity, diabetes, certain cancers and osteoporosis.

\section{References}

1. Becker W, Lyhne N, Pedersen AN, Aro A, Fogelholm M, Thórsdottír I, et al. Nordic Nutrition Recommendations 2004. Integrating nutrition and physical activity. Scand J Nutr 2004; 48: 178-87.

2. Becker W, Lyhne N, Pedersen AN, Aro A, Fogelholm M, Thórsdottír I, et al. Nordic Nutrition Recommendations 2004. Integrating nutrition and physical activity. Nord 2004:13. Nordic Council of Ministers, Copenhagen, 2004.

3. Enghardt Barbieri H, Lindvall C. Svenska Näringsrekommendationer översatta till livsmedel. Underlag till generella råd på livsmedelsnivå (Swedish Nutrition Recommendations translated into foods). Livsmedelsverket, Rapport 1-2003.

4. Tuomilehto J, Lindström J, Eriksson JG, Valle TT, Hämäläinen H, Ilanne-Parikka P, et al. Finnish Diabetes Prevention Study Group. Prevention of type 2 diabetes mellitus by changes in lifestyle among subjects with impaired glucose tolerance. N Engl J Med 2001; 344: $1343-50$

5. Mensink M, Blaak EE, Corpeleijn E, Saris WH, de Bruin TW, Feskens EJ. Lifestyle intervention according to general recommendations improves glucose tolerance. Obes Res 2003; 11: 1588-96. 
6. Sacks FM, Svetkey LP, Vollmer WM, Appel LJ, Bray GA, Harsha D, et al. Effects on blood pressure of reduced dietary sodium and the Dietary Approaches to Stop Hypertension (DASH) diet. DASH-Sodium Collaborative Research Group. N Engl J Med 2001; 344: 310 .

7. Sandström B, Marckmann P, Bindslev N. An eightmonth controlled study of a low-fat high-fibre diet: effects on blood-lipids and blood pressure in healthy young subjects. Eur J Clin Nutr 1992; 46: 95-109.

8. Marckmann P, Sandström B, Jespersen J. Low-fat, highfiber diet favorably affects several independent risk markers of ischemic heart disease: observations on blood lipids, coagulation, and fibrinolysis from a trial of middle-aged Danes. Am J Clin Nutr 1994; 59: 935-9.

9. Becker W. Kolhydratbantning - inte effektivare än konventionell diet och dessutom riskabel på sikt (Car- bohydrate dieting - no more effective than conventional diet and may confer long-term risks). Vår Föda 2003; 55(6): $22-5$.

10. Willett WC, Stampfer MJ. Rebuilding the food pyramid. Sci Am 2003; 288: 64-71.

11. Kromhout D, Keys A, Aravanis C, Buzina R, Fidanza F, Giampaoli S, et al. Food consumption patterns in the 1960s in seven countries. Am J Clin Nutr 1989; 49: 88994.

\section{Wulf Becker}

Information and Nutrition Department

Swedish National Food Administration

PO Box 622

SE-75 126 Uppsala, Sweden

E-mail:wulf.becker@slv.se 